\title{
Brote de Intoxicación Alimentaria por Salmonella Enteritidis en Hospital Nacional Zacamil marzo $2007^{1}$
}

\author{
Bud of Nourishing Poisoning by Salmonella \\ Entiritidis in Zacamil Hospital. March 2007
}

Barahona de Figueroa, Jeannette ${ }^{2}$

Zúñiga, Jorge ${ }^{3}$

Cruz, Claudia ${ }^{4}$

\section{Resumen}

$E$ ntre el 4 y 5 de marzo del 2007, en el Hospital Zacamil, fue identificado un brote de 37 personas con gastroenteritis aguda quienes habian participado en una fiesta familiar; fueron atendidos 24 pacientes, de los cuales 16 fueron hospitalizados. La ausencia de un agente etiológico y la cantidad de pacientes que consultaron con el mismo cuadro clínico, y que todos provenían de un mismo lugar presentó un reto a la investigación. El tipo de estudio fue observacional de cohorte retrospectivo. Criterios de inclusión: pacientes hospitalizados que cumplian con la definición de caso propuesta. Se recolectaron variables demográficas, clínicás y muestras fecales. Una vez identificado el agente causal se enviaron muestras fecales confirmatorias al laboratorio Central Max Bloch de San Salvador. Se calcularon tasas de ataque global, tasa de ataque clínica, tasa de ataque de casos confirmados por coprocultivo, $y$ tasa de ataque por alimento. Captura de datos en paquete Epi Info y excel. Entre los resultados se obtuvo que la tasa de ataque por alimento más elevada (100\%) fue para sándwich de pollo, tasa de ataque global del $100 \%$, tasa de ataque clínica del $64 \%$, tasa de ataque de casos confirmados por coprocultivo del $100 \%$, tasa de internamiento de $66 \%$. El patógeno que se aisló correspondió a Salmonella sp Poly A1Vi Grupo D1, en el 100\% de las muestras fecales. Mayor confirmación (fago tipificación) demostró la presencia de Salmonella Grupo D1, factores 1, 9 y 12 en el $100 \%$. Se concluyó que el brote se debió a Salmonella enteritidis 9,12 gm fagotipo 1; los resultados sugieren un brote de fuente común.

Palabras claves: brote, intoxicación alimentaria, Salmonella enteritidis.

\section{Abstract}

$B$ etween March 4th and 5th of the 2007 at Zacamil Hospital, a bud of 37 people, who had participated in a familiar celebration, appeared with severe gastroenteritis; 24 patients were taken care of, 16 of them were hospitalized. The absence of an etiology agent, the number of patients who consulted with the same, and the fact that all came from a same place, challenged an investigation. The type of study was observational of retrospective type. The Criteria of inclusion: hospitalized patients who fulfilled the propose definition of case. Demographic, clinical and feces samples were collected. Once identified, the causing agent confirming feces samples were sent to the Max Bloch Central laboratory in the city of San Salvador. Rates of global attack were calculated, also rates of clinical attack, rate of attack of cases confirmed by coprocultivated, and the rate of attack by food. Database captured in package Epi Info and excel. Among the results, it was obtained that the rate of attack by elevated food was more than $(100 \%)$ because of chicken sandwich. The global attack rate was $100 \%$, the clinical attack rate was $64 \%$, the attack rate of confirmed cases by coprocultivated was $100 \%$, and the rate of internship was $66 \%$. The pathogen isolated corresponded to Salmonella sp Poly A1-Vi D 1 Group, in 100\% of the feces samples. Greater confirmation (fago type) demonstrated 100\% by the presence of Salmonella DI Group, factors 1,9 and 12 in the $100 \%$. It was concluded that the bud was due to Salmonella enteritidis 9.12 GM fago type 1; the results suggest a bud of common source.

Key words: it appears, nourishing poisoning, Salmonella enteritidis.

\footnotetext{
'Trabajo presentado al Tercer Certamen de Investigación Científica UEES, 10 de julio 2007. Ganador del segundo lugar. Categoría DocenteAdministrativos.

${ }^{2}$ Doctora en Medicina, Pediatra. Docente UEES- Hospital Nacional Zacamil. E-mail: figueroal6@hotmail.com.

3.4 Colaboradores participantes del estudio Facultad de Medicina de la UEES, destacados en el Hospital Nacional Zacamil "Dr. Juan José Fernández", Mejicanos, El Salvador.
} 


\section{Introducción}

A nivel mundial las enfermedades diarreicas constituyen un problema de salud pública, tanto para las naciones desarrolladas como para los países en vías de desarrollo. Las condiciones socio-ambientales en El Salvador no han sido las óptimas para el goce de la salud y en los últimos años no se ha logrado, pese a los esfuerzos orientados en este sentido, a controlar su tendencia al ascenso.

La alta incidencia de los procesos infecciosos entéricos se dan acompañando a vacaciones, fiestas, celebraciones populares, cambios ambientales preferentemente de precipitación fluvial, escasez de agua y consumo de alimentos no seguros lo que evidencia el poco ejercicio de las medidas higiénicas preventivas, para cortar la transmisión incorporada al ciclo ano-mano-boca. Los elevados índices de morbi-mortalidad hacen que este tipo de patología constituya un motivo de especial interés tanto desde el punto de vista clínico como microbiológico.

Aunque la intoxicación alimentaria es relativamente rara en los Estados Unidos, afecta entre 60 y 80 millones de personas en todo el mundo cada año y ocasiona aproximadamente de 6 a 8 millones de muertes.

La intoxicación alimentaria puede afectar a una persona o puede presentarse como un brote en un grupo de personas que comieron el mismo alimento contaminado. Tiende a ocurrir en las salidas al campo, en las cafeterías de las escuelas y en las reuniones sociales, situaciones en las cuales se pueden manejar alimentos sin refrigerar durante períodos de tiempo prolongado o las técnicas de preparación de éstos no son higiénicas (American Medical Association y otros centros, 2001).

El Hospital Nacional Zacamil "Dr. Juan José Fernández" mantiene a manera de captura de información el registro permanente de los eventos gastrointestinales. El hospital vigila las tendencias de las patologías existentes y es así como se identificó un número importante de casos entre niños y adultos, de enfermedad diarreica que consultaron al hospital el día 4 y 5 de marzo del 2007 , y que provenían de una misma fiesta, presuntamente provocados por alimento, los casos en su mayoría requirieron hospitalización.

Ante dicho evento personal médico, el hospital decidió investigar las características clínicas, epidemiológicas y de laboratorio de un brote de enfermedad gastrointestinal que no constituía lo habitual, situación que podría volverse un problema de mayores dimensiones.

\section{Fundamento del estudio}

Se determina un brote importante de casos de diarrea en niños y adultos que provenian de una misma fiesta familiar, en marzo 2007 y que consultaron al Hospital
Zacamil, a lo cual se planteó ¿Cual esielagente etiológico causante de dicho brote y cual es là fuente que lo produce?

La intoxicación alimentaria es un padecimiento que resulta del consumo de'organismos o toxinas en alimentos contaminados; puede afectar a una persona o puede presentarse como un brote en un grupo de personas que comieron el mismo alimento contaminado. Afecta entre 60 y 80 millones de personas en todo el mundo cada año y ocasiona aproximadamente de 6 a 8 millones de muertes (American Medical Association y otros centros, 2001)

Los niños y las personas de edad tienen el mayor riesgo de intoxicación por alimentos. Los síntomas de los tipos de intoxicación alimentaria más comunes generalmente comienzan en un período de 2 a 6 horas después de ingerir el alimento responsable. Los síntomas pueden incluir: náuseas y vómitos, cólicos abdominales, diarrea, fiebre y escalofríos, cefalea. (American Medical Association y otros centros, 2001)

Los animales utilizados para la producción de carne, lácteos y huevos, frecuentemente son portadores de Salmonella. La mayoría de los casos de enfermedad se originan por un manejo inadecuado de alimentos contaminados en cocinas y restaurantes. Es un agente zoonótico de distribución universal. Se transmite por contacto directo o contaminación cruzada durante la manipulación, en el procesado de alimentos .(Farreras Valenti, 2000)

Salmonella es un género de bacterias que pertenece a la familia Enterobacteriaceae, formado por bacilos gramnegativos, anaerobios facultativos, con flagelos que rodean al microorganismo y no desarrolla cápsula ni espora. Son bacterias móviles que producen sulfuro de Hidrógeno $\left(\mathrm{H}_{2} \mathrm{~S}\right)$. Fermentan glucosa pero no lactosa. El género Salmonella, es de taxonomía dificil, conceptualmente se ha modificado en estos últimos años, por el aporte de estudios moleculares de homología de ADN que han clarificado el panorama taxonómico de las enterobacterias. (Brooks G.F; 2002)

Para la bacteriología clínica, Salmonella es un patógeno primario, anaerobio facultativo, algunos móviles y no fermentan la lactosa. Salmonella tiphy es la única serovariedad que no produce gas en la fermentación de los azúcares. Clásicamente se distinguían tres únicas especies patógenas primarias: Salmonella tiphy, Salmonella cholerae-suis y Salmonella enteritidis. A su vez, según la serotipificación de Kauffman y White, eran clasificadas en más de 2000 serotipos en base a los antígenos flagelares $\mathrm{H}$ (proteicos) y antígenos somáticos $\mathrm{O}$ (fracción polisacárida del lipopolisacárido bacilar). Salmonella tiphy posee además un antígeno de virulencia (Vi) (Alejandra Velilla; 2004).

El tratamiento taxonómico actual de Salmonella ha simplificado el espectro, reagrupando todas las cepas (patógenas o no) en dos únicas especies: Salmonella entérica y Salmonella bongori. Esta última (previamente subespecie V) no es patógena para el ser humano (Alejandra Velilla; 2004). 
Hay más de 2.435 serovariedades o serotipos (término que utilizaremos siguiendo las recomendaciones del CDC de Atlanta) de Salmonella, la mayoría pertenecientes a la subespecie I, en la que se encuentran Salmonella enteritidis y Salmonella typhimurium que son las más frecuentes en nuestro medio (Rodriguez DC; 1990).

Para reducir las infecciones humanas por S. enteritidis, los manipuladores de alimentos necesitan una formación intensiva en lo que atañe a la manipulación correcta de alimentos crudos. Los alimentos preparados y los huevos deben mantenerse refrigerados durante su distribución y almacenamiento. Es fundamental mantener un alto nivel de higiene en la cocina y proteger a los alimentos preparados de la contaminación de insectos, roedores u otros animales (Organización Mundial de la Salud, 1988).

\section{Justificación}

Entre el 4 y 5 de marzo del año 2007 se identifica un brote importante de casos de gastroenteritis aguda, ocurrido en Mejicanos, en personas invitadas a una fiesta familiar, asociado presuntamente a ingesta de alimentos, entre niños y adultos que acudieron al Hospital Zacamil, resultando en hospitalizaciones.

La ausencia de un agente etiológico temprano en el curso del brote y la cantidad de pacientes que consultaron con el mismo cuadro clínico, y que provenían de un mismo lugar presentó un reto a la investigación.

El departamento de Pediatría del Hospital Zacamil, inició una investigación, a fin de establecer la definición de caso, determinar la causa del brote, identificar el agente etiológico y sus modos de transmisión, para poder implementar medidas de control apropiadas, aliviar las preocupaciones de los pacientes y efectuar recomendaciones para prevenir la ocurrencia de otros eventos similares.

Objetivo general:

Describir y determinar las causas de un brote de enfermedad diarréica ocurrido en Mejicanos, Marzo 2007 , entre asistentes a una fiesta familiar hospitalizados en Hospital Zacamil.

Objetivo especifico:

- Lograr la tipificación el agente causante del brote.

- Describir la sintomatología presentada durante el brote.

- Identificar las características epidemiológicas de los casos

- Identificar la morbi-mortalidad atribuible al brote de intoxicación alimentaria.

- Determinar los procesos o prácticas que permitieron la intoxicación

- Implementar medidas de control apropiadas para evitar un problema de dimensiones mayores.

\section{Metodología de la investigación}

El 4 de marzo del año 2007, el Hospital Zacamil, identifica un brote de 37 casos de gastroenteritis aguda, ocurrido en Mejicanos en personas que participaron de una fiesta familiar y que estuvo relacionado al consumo de alimentos, de los cuales 24 pacientes entre niños y adultos consultaron y se atendieron, resultando en 16 hospitalizaciones.

Se puso en marcha la investigación epidemiológica, realizándose un estudio observacional de cohorte retrospectivo. Se recolectaron datos demográficos, clínicos y muestras fecales de 14 pacientes aún hospitalizados en el Hospital Nacional Zacamil, a través de realización de historia clínica y encuesta alimentaria, utilizando una hoja de recolección de datos, donde se incluyeron las siguientes variables: número de registro, edad, sexo, procedencia, fecha y hora de consulta, fecha de inicio de síntomas, lista de alimentos ingeridos, criterios clínicos presentados, duración de la enfermedad, complicaciones, tratamiento, y estancia hospitalaria.

Se estableció como definición de caso de intoxicación alimentaria la siguiente: "enfermedad gastrointestinal sintomática: diarrea (tres o más deposiciones líquidas en 24 horas) y/o vómito, en personas que asistieron a la fiesta el 4 de marzo 2007, o que ingirieron alimentos provenientes de la misma, presentando síntomas dentro de un plazo de 72 horas siguientes al consumo de alimentos y que consultan al hospital Zacamil"

Caso confirmado: Aquel paciente que cumpla con la definición de caso y presente el hallazgo de determinado germen en el coprocultivo, alimento o materia prima con que fue elaborado.

Los criterios de inclusión: pacientes que permanezcan hospitalizados al momento de iniciar el estudio, que cumplan con la definición de caso propuesta. Criterios de exclusión: pacientes que no cumplieron con los criterios de definición de caso, pacientes de manejo ambulatorio, y los pacientes que se habían dado de alta antes de iniciar el estudio.

Análisis de muestras fecales: examen general de heces, prueba de azul de metileno y coprocultivo de los niños y adultos hospitalizados en busca de virus, bacterias, y parásitos. Se utilizaron medios de cultivo diferencial: Agar MacConkey, SS, TCBS, caldo selenito.

Una vez demostrado el agente causal del brote por el laboratorio del Hospital Zacamil, se enviaron muestras al laboratorio Central Max Bloch para pruebas confirmatorias y tipificación final respectiva. Captura de datos y análisis estadístico paquete informático Epi info, y Excel.

Se calcularon tasas de ataque global, tasa de ataque clínica, tasa de ataque de internamiento, tasa de ataque de casos confirmados por coprocultivo en los investigados, y tasa de ataque para cada alimento del menú de la fiesta. Dado que no se pudo entrevistar 
a otros asistentes a la fiesta no fue suficiente la información obtenida sólo de los pacientes enfermos para efectuar análisis de estimación de riesgos en función al consumo de alimentos. Consideraciones éticas fueron tomadas al momento de la investigación por ûn lado la obtención del consentimiento informado de su participación en el estudio, y por otra el respeto a los principios de la investigación biomédica principalmente referente a la seguridad y la confidencialidad en el uso y manejo de las muestras y los datos asociados con ellas.

\section{Análisis y discusión de resultados}

\section{Investigación epidemiológica.}

\begin{tabular}{|c|c|c|c|c|}
\hline Casos & $\begin{array}{l}N^{\circ} \text { Total de } \\
\text { Personas que } \\
\text { Enfermaron }\end{array}$ & $\begin{array}{l}N^{\circ} \text { Total de } \\
\text { Personas que } \\
\text { Consultaron }\end{array}$ & $\begin{array}{l}\mathrm{N}^{0} \text { de Paciente } \\
\text { Ingresados }\end{array}$ & $\begin{array}{l}N^{0} \text { Total de } \\
\text { Pacientes Incluidos } \\
\text { en el Estudio }\end{array}$ \\
\hline 37 & 37 & 24 & 16 & 14 \\
\hline $\begin{array}{c}\text { Total } \\
37\end{array}$ & $\begin{array}{l}\text { Tasa de ataque } \\
\text { global } 100 \%\end{array}$ & $\begin{array}{l}\text { Tasa de ataque } \\
\text { clínica } \\
64 \%\end{array}$ & $\begin{array}{c}\text { Tasa de ataque } \\
\text { de internamiento } \\
66 \%\end{array}$ & Muestra 14 \\
\hline
\end{tabular}

Se declararon un total de 37 casos que presentaban sintomatología de enfermedad gastrointestinal y que habían consumido alimentos repartidos en una fiesta en Mejicanos el 4 de marzo 2007, de los cuales consultaron al Hospital 24 casos, el 4 de marzo: 17 casos y el 5 de marzo: 7 casos, todos respondían a los criterios de definición de caso establecidos, hospitalizándose 16 de ellos y manejándose 8 casos de manera ambulatoria.

Se incluyeron en el estudio 14 casos de los 16 que permanecían hospitalizados al momento de la investigación la cual se inició 2 días después del ingreso de los pacientes.

La tasa de ataque global de las personas expuestas fue de $100 \%$, todos los pacientes encuestados que dijeron haber comido sándwiches de pollo enfermaron, la tasa de ataque clínica fue de $64 \%, 24$ que respondieron a la definición de caso consultaron al hospital, de 37 personas que asistieron a la fiesta y enfermaron. La tasa de internamiento de los pacientes que consultaron fue del $66 \%$.

\section{Caracterización de los casos estudiados}

En el estudio estuvieron involucrados 8 casos del género femenino y 6 del masculino; los rangos de edad oscilaron de 1 a 75 años de los cuales 8 eran niños y 6 adultos. La procedencia de los pacientes fue 12 de Mejicanos y 2 de Cuscatancingo.

Los 14 pacientes tenían en común el haber asistido a una fiesta familiar donde habían ingerido sándwich de pollo (el único alimento que habían comido el $100 \%$ de los pacientes), 7 pacientes comieron pastel, 12 pacientes tomaron horchata y 6 pacientes tomaron gaseosa.
La tasa de ataque por alimento según la encuesta alimentaria, para los pacientes ingresados fue de $100 \%$ para sándwich de pollo, $50 \%$ para el pastel, $78 \%$ para la horchata, y $35 \%$ para la gaseosa, como se observa en el gráfico 1 y cuadro 2.

\section{Gráfico 1. Tas a de ataque por alimento}

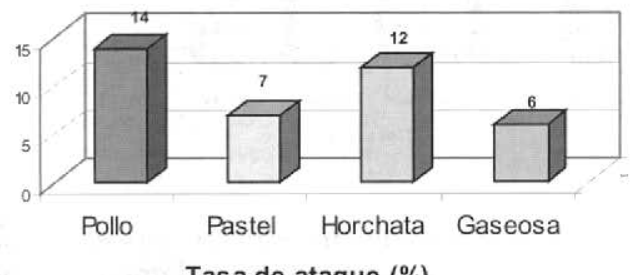

Tasa de ataque $(\%)$

\begin{tabular}{|c|c|c|c|c|}
\hline Pacientes & Pollo & Pastel & Horchata & Gaseosa \\
\hline 1 & $\mathrm{Si}$ & Si & $\mathrm{Si}$ & si \\
\hline 2 & Si & Si & $\mathrm{Si}$ & si \\
\hline 3 & $\mathrm{Si}$ & Si & Si & si \\
\hline 4 & $\mathrm{Si}$ & Si & Si & si \\
\hline 5 & $\mathrm{Si}$ & Si & Si & si \\
\hline 6 & Si & Si & $\mathrm{Si}$ & . \\
\hline 7 & Si & Si & Si & - \\
\hline 8 & Si & - & Si & - \\
\hline 9 & $\mathrm{Si}$ & - & Si & - \\
\hline 10 & $\mathrm{Si}$ & - & Si & - \\
\hline 11 & Si & - & Si & - \\
\hline 12 & Si & - & - & - \\
\hline 13 & Si & - & - & - \\
\hline 14 & $\mathrm{Si}$ & - & - & - \\
\hline Total & 14 & 7 & 12 & 6 \\
\hline $\begin{array}{l}\text { Tasa de } \\
\text { ataque \% }\end{array}$ & 100 & 50 & 78 & 35 \\
\hline
\end{tabular}

La hora de consumo de los sándwich de pollo osciló entre $4 \mathrm{pm}$. y las $8 \mathrm{pm}$. Con una mediana de las 4 pm. Fueron 3 pacientes los que comieron entre las 7 y $8 \mathrm{pm}$. ya que no asistieron a la fiesta pero les mandaron sándwiches a sus casas.

La hora de inicio de los síntomas osciló entre 3 y 15 horas después de haber comido sándwich de pollo. Con una mediana de 4.8 horas. Los 3 pacientes que ingirieron tardíamente los sándwiches dieron las manifestaciones clínicas más tardías (Gráfico 2).

Gráfico 2. Distribución de casos por hora de inicio de síntomas

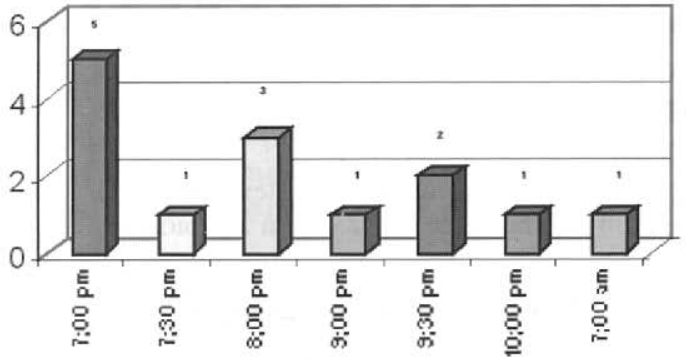

Los síntomas se caracterizaron por su pronta aparición (entre tres y 15 horas), y fueron diarrea y vómitos en 14 casos $(100 \%)$, dolor abdominal en 13 pacientes $(92 \%)$, fiebre en 11 casos, $(78 \%)$ y cefalea en 2 casos (14\%) (Gráfico 3). 
Gráfico 3. Distribución de casos según síntomas

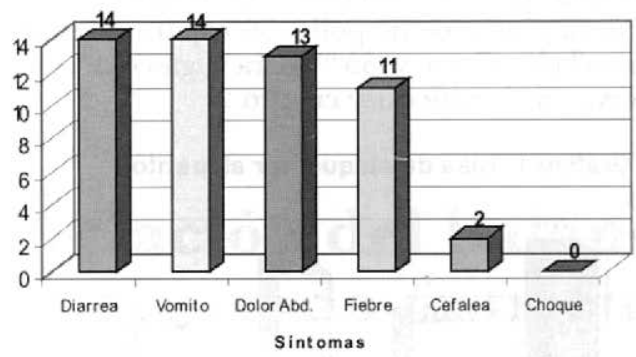

Con el diagnóstico de ingreso: 10 casos fueron diagnosticados a su ingreso apropiadamente como intoxicación alimentaria, 4 como síndrome diarreico agudo, posteriormente a su apropiada identificación y en respuesta al cumplimiento de la definición de caso se modificó el diagnóstico.

\section{Investigación de laboratorio}

En el Laboratorio 11 pacientes presentaron leucocitosis fecal y tuvieron la prueba de azul de metileno positiva a polimorfonucleares y 3 prueba de azul de metileno negativo.

Mayor caracterización fue realizada por el Laboratorio Central Dr. Max Bloch, demostró que de 13 muestras fecales que les fueron enviadas para análisis, fueron tipificadas con antisueros hasta llegar a clasificarlas como Salmonellas del grupo Dl factores 1,9 y 12 , faltando los antisueros de la fase flagelar que en ese momento se habían agotado, posteriormente y con el apoyo de antisueros provenientes de una reciente dotación se completa la serotipificación final a Salmonella enteritidis, cuya fórmula antigénica es 1, 9, $12 \mathrm{gm}$. en los 13 pacientes enviados, demostrando una tasa de ataque de casos confirmados por coprocultivo del $100 \%$.

Tabla 3. Resultados de Laboratorio

Laboratorio Hospital Laboratorio Central Dr. Max Bloch,

Zacamil. (13 muestras) (13 muestras confirmadas por Hospital)

Salmonella sp Poly A1-Vi Salmonella Grupo D1, Factores 1, Grupo D1.

\section{Tratamientos de pacientes}

El tratamiento recibido: 8 pacientes fueron manejados con Ampicilina intravenosa y 6 sin antibiótico (Gráfico 4).

Gráfico 4. Tratamiento en pacientes

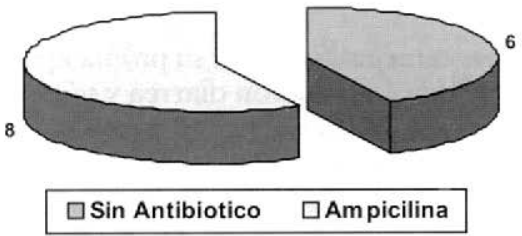

Se realizó antibiograma respectivo con discos existentes demostrando un patrón de sensibilidad a: Ampicilina, Cloranfenicol, Ceftazidima, Cefotaxima.

Los pacientes mostraron una recuperación completa a los 2 a 3 días después de haber iniciado el cuadro. El promedio de estancia hospitalaria fue de 3 días. No hubo complicaciones en los pacientes hospitalizados, ni letalidad reportada.

\section{Investigación ambiental}

El SIBASI Centro a través del área de epidemiología, identificó algunos factores de riesgo que podrían haber influido en el surgimiento de este brote por transmisión de alimentos: que el pollo con el que se habían preparado los sándwiches se había comprado en un mercado local, "la tiendona", un día antes de la fiesta, pero no pudo establecerse si el comercio había mantenido el producto en refrigeración, que según las condiciones de los mercados del país, lo más probable es que se mantuvo a temperatura ambiente. Al día siguiente de la compra, el pollo se había preparado en casa y lo habían desecho con las manos (una de las personas que también enfermó) y se había mantenido a temperatura ambiente, por 8 horas desde su preparación hasta su consumo.

Los sándwiches de pollo no pudieron ser recuperados para investigación microbiológica ya que la investigación se inició 48 horas, después de pasada la fiesta.

Todas las personas que comieron sándwich de pollo enfermaron. Investigación adicional demostró que 3 pacientes que no habían asistido a la fiesta y que sus familiares les habían llevado sándwich repartidos en la misma, ingirieron los sándwich entre las 7 y 8 pm también enfermaron y son los que más tardaron en iniciar los síntomas 5.5, 6 y 15 horas posterior a la ingesta.

\section{Discusión}

La presencia del brote diarreico ocurrido en Mejicanos en pacientes que provenían de un fiesta familiar en marzo 2007, evidenció una exposición amplia en relación con los alimentos distribuidos en dicha fiesta. Este brote es importante, por el hecho de que toda la población expuesta al alimento sándwich de pollo enfermó en un $100 \%$ y se determinó en el $100 \%$ de los casos el agente etiológico, verificándose con esto la alta contaminación, y pobres medidas higiénicas de preparación y de conservación de alimentos.

La identificación de Salmonella enteritidis aislada en el $100 \%$ de las muestras fecales examinadas, determinó que era el agente causal del brote de intoxicación alimentaria. El análisis sintomático de los afectados evidenció que estaba acorde a la sintomatología presentada por el agente etiológico identificado por el laboratorio de referencia en las muestras procesadas. 
Tomando en cuenta la hora de distribución de los sándwiches de pollo y la hora de inicio de los síntomas de la mayoría de los sujetos afectados, resulta altamente probable que la fuente de contaminación identificada fuesen los sándwiches de pollo, ya que el período trascurrido entre la ingesta y la hora de inicio de los síntomas de la mayoría de la población no supera el periodo de incubación de la bacteria reportada por el laboratorio sugiriendo con esto un brote de fuente común.

En relación a la preparación del alimento contaminado un factor importante en el origen del brote podría ser que el pollo con el que fueron preparados los sándwiches, ya que fue comprado en el mercado local "La Tiendona". No se pudo establecer que el comercio había mantenido el pollo en refrigeración, el pollo se había preparado en casa y lo habían desecho con las manos y se había mantenido a temperatura ambiente, por 8 horas hasta su consumo lo que evidencia las condiciones necesarias para la transmisión del agente aislado.

Considerando el período de incubación de la enfermedad producida por el Salmonella enteritidis, el tipo de alimento brindado y la hora en la que fue distribuido, aunado a la sintomatología asociada y las condiciones higiénicas del país en relación a la contaminación de carnes y sus derivados, los resultados son concordantes con la identificación etiológica brindada por el Laboratorio Central "Dr. Max Bloch", que fue tomado como referente.

Es importante establecer un control más estricto en el procesamiento de los productos avícolas tanto en el ámbito de las granjas destinadas para este propósito como en la preparación de los alimentos. Los brotes de infección por Salmonella enteritidis constituyen un problema de salud pública en El Salvador y otros países. Se ha aislado de las aves, de huevos contaminados, o con cascaron resquebrajado.

En esta investigación queda demostrado que Salmonella enteritidis es una enfermedad zoonótica que causa brotes en el país por lo que cobra importancia para la salud pública del mismo ya que podría ocasionar problemas de mayores dimensiones debido a que se transmite a través de la ingesta de productos de consumo masivo como son el pollo o huevos, y que por lo tanto deben implementarse medidas de control y vigilancia más estrictas de las granjas avícolas por las autoridades pertinentes.

\section{Conclusiones}

- La investigación demostró que Salmonella enteritidis $9,12 \mathrm{gm}$. fagotipo 1 fue el agente infeccioso causante del brote de intoxicación alimentaria en marzo 2007 en personas provenientes de una fiesta en Mejicanos, ya que se aisló y tipificó en el $100 \%$ de todas las muestras fecales analizadas;

- Las principales características clínicas presentadas en este brote fueron: vómito, diarrea, dolor abdominal y fiebre principalmente;

- Investigación epidemiológica sugiere que los sándwiches de pollo fueron el alimento contaminado que causó el brote de intoxicación;

- Debido a la ocurrencia de todos los casos en tiempo corto y su presentación dentro del período de incubación sugiere que fue un brote de fuente común;

- La notificación obligatoria por parte del área clínico- hospitalaria a las autoridades pertinentes a su investigación, no fue inmediata lo que dió lugar a que la investigación ambiental de este brote no fuera la ideal;

- No se llevó a cabo ningún estudio de muestras ambientales;

- El informe sanitario ambiental sugiere que la manipulación y conservación inadecuada de los alimentos fue un factor importante que contribuyó a la alta tasa de ataque mostrada por los sándwiches de pollo durante el brote;

- El aislamiento de Salmonella enteritidis en un brote relacionado con alimentos ha sido poco frecuente en nuestro medio, y su caracterización cobra importancia ya que traduce que debe monitorizarse la cadena de producción avícola, en puntos de control críticos para detectar si ha habido contaminación por Salmonella y así poder adoptar las medidas necesarias para eliminar el organismo tras detectarse la contaminación.

\section{Recomendaciones}

- Capacitar a los médicos residentes en cuanto a vigilancia epidemiológica para hacer efectiva la notificación inmediata de este tipo de brotes a las autoridades pertinentes y lograr una mejor caracterización, control y aplicación de medidas preventivas apropiadas;

- Para mejorar las técnicas de vigilancia y facilitar la investigación de brotes recomendamos que en el país se fortalezca el área de microbiología para la sistematización de la identificación de cepas de agentes entero patógenos;

- Continuar con el fortalecimiento de la alianza entre el Hospital y otras entidades de cooperación en este caso Laboratorio Central Max Bloch;

- Fomentar la promoción de la salud, la higiene, buen tratamiento térmico y buena conservación de los alimentos dirigida al grupo expuesto, para no verse afectados por nuevos episodios de enfermedad gastrointestinal y a la población en general;

- Controlar y dar seguimiento a los pacientes del brote con la finalidad de evitar el estado de portador y así poder evitar futuras diseminaciones;

- Fortalecer la comunicación de este tipo de brotes por parte del Ministerio de Salud Pública al Ministerio de Agricultura y Ganadería para la toma de medidas de control sanitario. 


\section{Fuentes de información consultadas}

1. American Medical Association, Centers for Disease Control and Prevention, Center for Food Safety and Applied Nutrition, Food and Drug Administration, Food Safety and Inspection Service, US Department of Agriculture. Diagnosis and management of foodborne illnesses: a primer for physicians. Atlanta, Ga. 2001. MMWR Recomm Rep; 50(RR-2): 1-69.

2. Farreras Valentí $P$ \& Rozman C (et al.): Medicina Interna, Harcourt, 2000.

3. Rodriguez DC, Tauxe RV, Rowe B. International increase in Salmonella enteritidis: A new pandemic? Epidemiol Infect 1990;105:21-27.

4. Organización Mundial de la Salud. Control de la salmonelosis: importancia de la higiene veterinaria y de los productos de origen animal. Informe de un comité de expertos de la OMS. Ginebra: OMS, 1988; (Serie de Informes Técnicos núm. 774).

5. Alejandra Velilla, Dr. Horacio Terzolo \& Dr. Sergio Feingold. Avances en el diagnóstico molecular de Salmonella:2004

6. Brooks G. F., 7. Butel and S. Morse. Microbiología Medica de Jawetz, Melnicky Adelberg, $17^{\circ}$ edición. El Manual Moderno, S. A. De C. V., 2002. Bacilos Gram negativos, Salmonella y Shiguella.

7. Mancini S, Bigatti R. (1998). Estudio de un brote de salmonellosis humana producido por la ingestión de helado contaminado en Viedma, Río Negro. En resumen $2^{\circ}$ Congreso Argentino de Zoonosis. Buenos Aires, pág 186.

8. Kassa H, Harrington B, Bisesi M, Khuder S. (2001). Comparisons of microbiological evaluations of selected kitchen with visual inspections for preventing potential risk of foodborne outbreaks in food service operations. 7 Food Prot., 64: 509-13.

9. Parrilla Cerrillo $M C$, Vázquez Castellanos $7 L$, Saldate Castañeda EO, Nava Fernández LM. (1993). Brotes de toxiinfecciones alimentarias de origen microbiano $y$ parasitario. Salud Pública México, 35: 456-63.

10. Grillo Rodríguez M, Lengomín Fernández M, Caballero Torres A, Castro Dominguez A, Hernández Alvarez AM. (1996). Análisis de las enfermedades transmitidas por los alimentos en Cuba. Revista Cubana Aliment Nutr.

11. Van Loock F, Ducoffre G, Dumont FM, et al. (2000). Analysis of foodborne disease in Belgium in 1997. Acta Clin Belg, 55: 300-6.

12. Bonner C, Foley B, Wall P, Fitzegarld M. (2001). Analysis of outbreaks of infectious intestinal disease in Ireland 1999. Ir Med 7. 94:142-4.

13 Olsen S, MacKinon L, Goulding J, Bean N, Slutsker L. $C D C$. (2000). Surveillance for foodborne disease outbreaks, USA, 1993-1997. MMWR, 1: 51.

14. Hennessy TW, Hedberg CW, Slutsker L. (1996). A national outbreak of Salmonella enteritidis infections from ice cream. $\mathcal{N}$ Engl 7 Med . 334: 1281-6.

15. Palmer S, Parry S, Perry D, et al. (2000). The role of outbreaks in developing food safety policy: population based surveillance of Salmonella outbreaks in Wales 19861998. Epidemiol Infect, 125: 467-72.

16. Harrison C, Quigley C, Kaczmarski E, Devlin E. (1992). An outbreak of gastro-intestinal illness caused by eggs containing Salmonella enteritidis phage type 4. 7 Infect. 24: $207-10$.

17. Lin FY, Morris $7 G$ Fr, Trump D, Tilghman D, Wood $P K$, fackman $\mathcal{N}$, et al. (1988). Investigation of an outbreak of Salmonella enteritidis gastroenteritis associated with consumption of eggs in a restaurant chain in Maryland. Am ff Epidemiol . 128: 839-44.

18. Thong KL, Puthucheary S, Pang T. (1998). Outbreak of Salmonella enteritidis gastroenteritis: investigation by pulsed-field gel electrophoresis. Int 7 Infect Dis. 2: 159 63.

19. Buchner $L$, Wermter $R$, Henkel $S$. Salmonella enteritidis in chicken eggs. Berl Munch Tierarztl Wochenschr. 